\title{
Visualizable and interpretable regression models with good prediction power
}

\author{
HYUNJOONG KIM ${ }^{1}$, WEI-YIN LOH ${ }^{2, *}$, YU-SHAN SHIH ${ }^{3}$ and PROBAL CHAUDHURI ${ }^{4}$ \\ ${ }^{1}$ Department of Applied Statistics, Yonsei University, 134 Shinchon-dong, Seodaemun-gu, Seoul 120-749, Korea \\ ${ }^{2}$ Department of Statistics, University of Wisconsin, 1300 University Avenue, Madison, WI 53706, USA \\ E-mail:loh@stat.wisc.edu \\ ${ }^{3}$ Department of Mathematics, National Chung Cheng University, Minghsiung Chiayi 621, Taiwan ROC \\ ${ }^{4}$ Division of Theoretical Statistics \& Mathematics, Indian Statistical Institute, Calcutta 700035, India
}

Received July 2005 and accepted January 2006

\begin{abstract}
Many methods can fit models with a higher prediction accuracy, on average, than the least squares linear regression technique. But the models, including linear regression, are typically impossible to interpret or visualize. We describe a tree-structured method that fits a simple but nontrivial model to each partition of the variable space. This ensures that each piece of the fitted regression function can be visualized with a graph or a contour plot. For maximum interpretability, our models are constructed with negligible variable selection bias and the tree structures are much more compact than piecewise-constant regression trees. We demonstrate, by means of a large empirical study involving 27 methods, that the average prediction accuracy of our models is almost as high as that of the most accurate "black-box" methods from the statistics and machine learning literature.
\end{abstract}

Keywords: Machine learning, piecewise linear, regression tree, selection bias

\section{Introduction}

Box (1979) wrote, "All models are wrong but some are useful". This statement is unquestionably true, but it raises the question: useful for what? There are two ways in which a model can be useful: (i) it can improve our understanding of the system generating the data; or (ii) it can make accurate predictions of future observations. For example, linear models for designed factorial experiments are useful because the terms they contain may be interpreted as main and interaction effects. On the other hand, accurate weather prediction models are useful even if they are hard to interpret.

There are many applications, however, where traditional statistical models are useless for prediction and for interpretation. An example is the study on house prices in the greater Boston area in 1970 reported in Harrison and Rubinfeld (1978) and made famous by Belsley et al. (1980). There are 506 observations on a variety of variables, with each observation pertaining to one census tract. The goal of the study was to build a regression model for the median house price (MEDV) and to use it to estimate the "marginal-willingnessto-pay for clean air," namely, the effect of nitrogen oxide

*Corresponding author concentration (NOX). Table 1 lists the predictor variables. After transforming some variables to satisfy normal-theory assumptions, Harrison and Rubinfeld (1978) obtained the fitted model shown in Table 2. Note that because the whole population is represented in the data, there is nothing to predict. In particular, the $t$-statistics do not have their usual statistical meaning.

We may hope that the model can explain the effects of the predictor variables on the response. For example, the sign associated with the coefficient for $\mathrm{NOX}^{2}$ suggests that it has a negative effect on MEDV. Similarly, the negative coefficient for $\log$ (DIS) leads to the conclusion that MEDV is negatively associated with DIS. Table 2 shows, however, that the correlation between $\log$ (DIS) and $\log$ (MEDV) is positive! Another example is RAD, which has a positive regression coefficient but a negative correlation with MEDV. Of course, these apparent contradictions are easy to explain. First, a regression coefficient quantifies the residual effect of the predictor after the linear effects of the other predictors in the model have been accounted for. Second, the correlation between a predictor and the response measures their linear association, ignoring the other predictors. Nevertheless, the contradictions in signs are not intuitive.

Can we construct models that are more interpretable and that also fit the data well? Since a model that involves a single predictor variable is easiest to interpret because the 
Table 1. Variables in the Boston housing data

\begin{tabular}{ll}
\hline Var & \multicolumn{1}{c}{ Definition } \\
\hline MEDV & Median value in $\$ 1000$ \\
DIS & Distance to employment centers \\
RAD & Accessibility to radial highways \\
INDUS & $\%$ nonretail business \\
CHAS & One if on Charles River, zero else \\
NOX & Nitrogen oxide conc. $\left(\mathrm{p} . \mathrm{p} .10^{9}\right)$ \\
RM & Average number of rooms \\
AGE & $\%$ built before 1940 \\
CRIM & Per capita crime rate \\
ZN & $\%$ land zoned for lots \\
TAX & Property tax $/ \$ 10,000$ \\
PT & Pupil/teacher ratio \\
B & $(\% \text { black }-63)^{2} / 10$ \\
LSTAT & $\%$ lower-status pop. \\
\hline
\end{tabular}

fitted function can be graphed, one solution is to employ the best single-predictor model. Unfortunately, because such a model does not incorporate the information contained in the other predictors, it may not fit the data as well as a model that uses more than one predictor. Furthermore, a singlepredictor model reveals nothing about the joint effect of all the predictors.

The goal of this paper is to study an alternative approach that: (i) retains the clarity and ease of interpretation of relatively simple models; (ii) allows expression of the joint effect of several predictors; and (iii) yields models with a higher average prediction accuracy than the traditional multiple linear regression model. We accomplish this by fitting simple-models to partitions of the dataset and sample space. One such model for the Boston data is shown by the tree structure in Fig. 1. The predictor space is split into three rectangular partitions. Within each partition, the best single predictor variable is selected to fit a linear model to MEDV. Notice that, unlike the Harrison-Rubinfeld model,

Table 2. Least squares fit for $\log (\mathrm{MEDV})$. The columns labeled by $\beta$, $t$, and $\rho$ give the estimated regression coefficients, $t$-statistics, and correlation between $\log (\mathrm{MEDV})$ and the corresponding $X$ variable

\begin{tabular}{lcrr}
\hline$X$ & \multicolumn{1}{c}{$t$} & \multicolumn{1}{c}{$\rho$} \\
\hline Intercept & 4.6 & 29.5 & \\
$\log ($ LSTAT) & $-3.7 \mathrm{E}-1$ & -14.8 & -0.8 \\
CRIM & $-1.2 \mathrm{E}-2$ & -9.5 & -0.5 \\
PT & $-3.1 \mathrm{E}-2$ & -6.2 & -0.5 \\
$\log ($ DIS $)$ & $-1.9 \mathrm{E}-1$ & -5.7 & 0.4 \\
NOX & $-6.4 \mathrm{E}-1$ & -5.6 & -0.5 \\
$\log ($ RAD $)$ & $9.6 \mathrm{E}-2$ & 5.0 & -0.4 \\
RM & $6.3 \mathrm{E}-3$ & 4.8 & 0.6 \\
B & $3.6 \mathrm{E}-4$ & 3.5 & 0.4 \\
TAX & $-4.2 \mathrm{E}-4$ & -3.4 & -0.6 \\
CHAS & $9.1 \mathrm{E}-2$ & 2.8 & 0.2 \\
AGE & $9.1 \mathrm{E}-5$ & 0.2 & -0.5 \\
ZN & $8.0 \mathrm{E}-5$ & 0.2 & 0.4 \\
INDUS & $2.4 \mathrm{E}-4$ & 0.1 & -0.5 \\
\hline
\end{tabular}

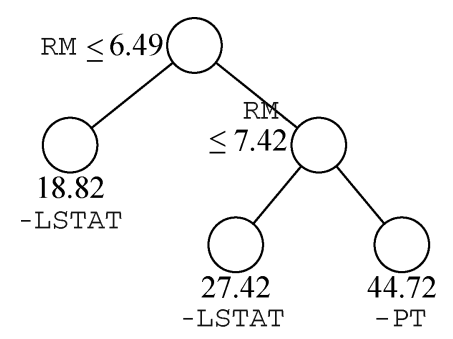

Fig. 1. Piecewise simple linear regression tree for the Boston data. The sample mean MEDV value and the best linear predictor is printed beneath each leaf node, together with the sign of its coefficient. At each split, a case goes down the left branch if and only if the associated inequality is satisfied.

we can directly model MEDV in terms of the original predictors without needing any transformations.

Figure 2 displays the data and fitted functions in the three partitions. The graphs indicate that LSTAT has a large negative effect on house price, except in census tracts with large houses (right panel) where PT is a stronger linear predictor. As expected, MEDV tends to increase with RM. These conclusions are consistent with the signs of the coefficients of $\log$ (LSTAT) and RM ${ }^{2}$ in the Harrison-Rubinfeld model.

Besides a piecewise single-regressor model, a piecewise two-regressor model can also be used to reveal more insight into the data. The tree structure for the latter is presented in Fig. 3, with the selected regressors printed beneath the leaf nodes. By utilizing only two regressor variables in each node of the tree, we can employ shaded contour plots to display the fitted functions and the data points. These plots are shown in Fig. 4, with lighter shades corresponding to higher values of MEDV. Note that some of the contour lines are not parallel; this is due to truncation of the predicted values, as explained by the algorithm in Section 2. We observe that the higher-priced census tracts tend to have high values of RM and low values of LSTAT. The lowest-priced tracts are mostly concentrated in one leaf node (bottom left panel in dark gray) with below average values of RM and DIS, and above average values of RAD, LSTAT, and CRIM. Although the regression coefficients in each leaf node model suffer from the problems of interpretation noted earlier, we do not need their values for a qualitative analysis. The contour plots convey all the essential information.

How well do the tree models fit the data compared to the Harrison-Rubinfeld model? Figure 5 plots the fitted versus observed values of MEDV. The piecewise two-regressor model clearly fits best of all. Notice the lines of points on the right edges of the graphs for the Harrison-Rubinfeld and the one-regressor tree models. They are due to the observed MEDV values being truncated at $\$ 50000$ (Gilley and Pace, 1996) and the inability of these two models to fit them satisfactorily. Our two-regressor model has no trouble with these points.

The rest of this article is organized as follows. Section 2 describes our regression tree algorithm. Section 3 analyzes 

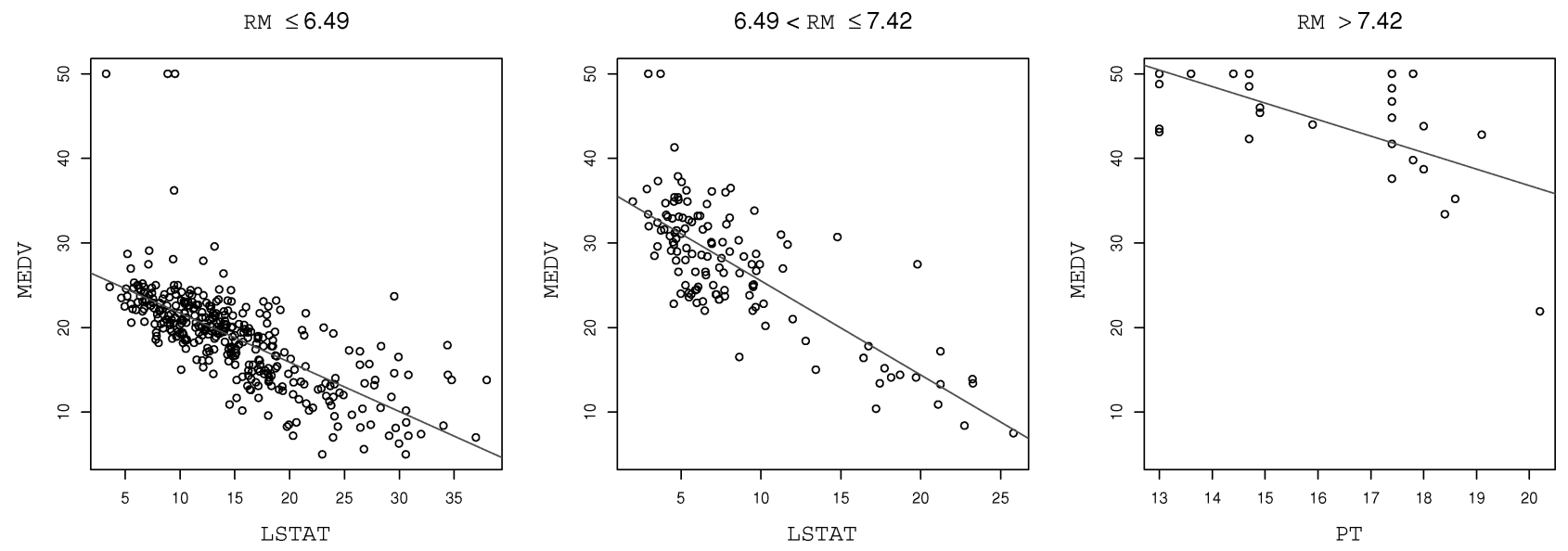

Fig. 2. Data and fitted models in three leaf nodes of the tree in Fig. 1.

another well-known dataset and compares the results with that of human experts. We take the opportunity there to highlight the important problem of selection bias. In Section 4 we compare the prediction accuracy of 27 algorithms from the statistical and machine learning literature on 52 real datasets. The results show that some machine learning methods have very good accuracy and that our methods are quite competitive. We prove an asymptotic consistency result in Section 5 to lend theoretical support to the empirical findings and close with some remarks in Section 6.

\section{Regression tree method}

Our algorithm is an extension of the GUIDE algorithm (Loh, 2002), which fits a constant or a multiple linear model at each node of a tree. The only difference is that we now use stepwise linear regression instead. The number of linear predictors permitted at each node may be restricted

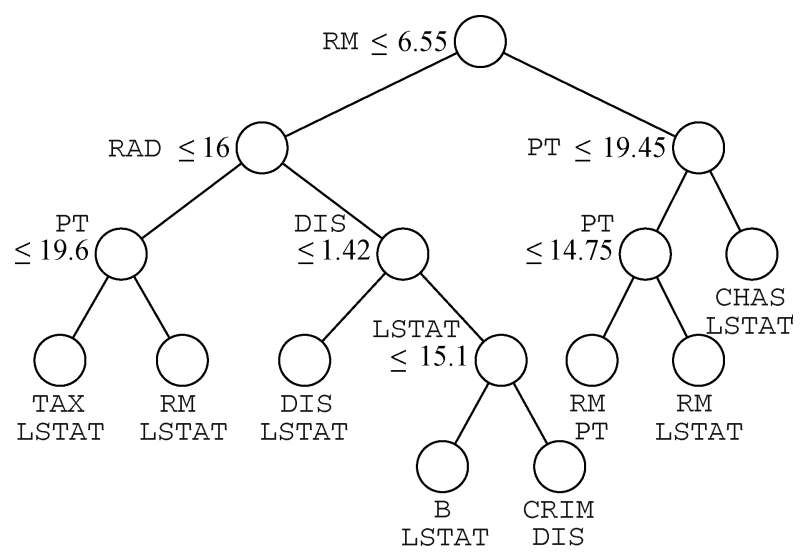

Fig. 3. Piecewise two-regressor linear regression tree for MEDV (Boston data), with selected regressor variables beneath each leaf node. or unrestricted, subject to the standard F-to-enter and F-to-remove thresholds of 4.0 (Miller, 2002). A one- or tworegressor tree model is obtained by restricting the number of linear predictors to one or two, respectively. We present here the recursive sequence of operations for a two-regressor tree model; the method for a one-regressor tree model is similar.

Step 1. Let $t$ denote the current node. Use stepwise regression to choose two quantitative predictor variables to fit a linear model to the data in $t$.

Step 2. Do not split a node if its model $R^{2}>0.99$ or if the number of observations is less than $2 n_{0}$, where $n_{0}$ is a small user-specified constant. Otherwise, go to the next step.

Step 3. For each observation, define the class variable $Z=$ 1 if it is associated with a positive residual. Otherwise, define $Z=0$.

Step 4. For each predictor variable $X$ :

(a) Construct a $2 \times m$ cross-classification table. The rows of the table are formed by the values of $Z$. If $X$ is a categorical variable, its values define the columns, i.e., $m$ is the number of distinct values of $X$. If $X$ is quantitative, its values are grouped into four intervals at the sample quartiles and the groups constitute the columns, i.e., $m=4$.

(b) Compute the significance probability of the chi-squared test of association between the rows and columns of the table.

Step 5. Select the $X$ with the smallest significance probability to split $t$. Let $t_{\mathrm{L}}$ and $t_{\mathrm{R}}$ denote the left and right subnodes of $t$.

(a) If $X$ is quantitative, search for a split of the form $X \leq x$. For each $x$ such that $t_{\mathrm{L}}$ and $t_{\mathrm{R}}$ each contains at least $n_{0}$ observations:

i. Use stepwise regression to choose two quantitative predictor variables to fit a 

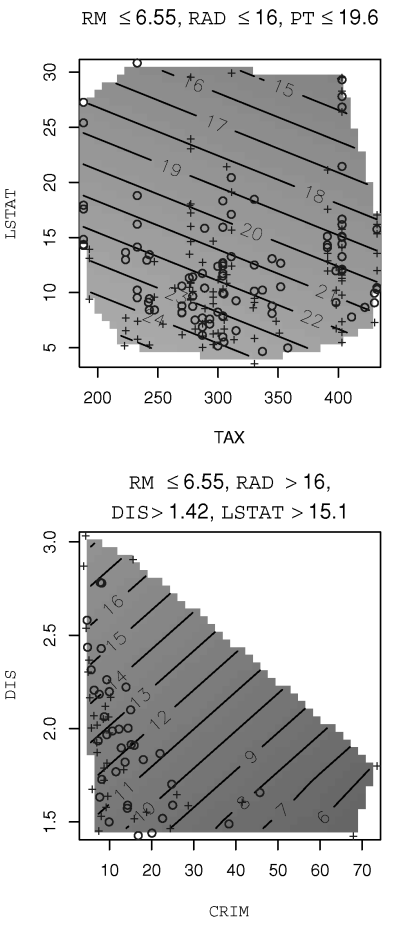

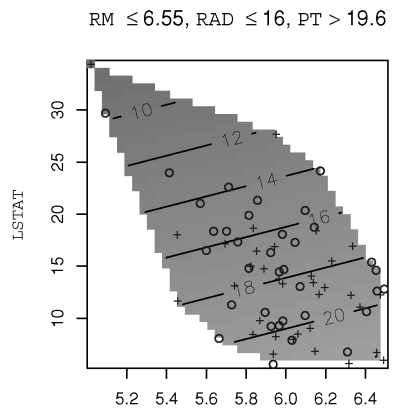

RM

$\mathrm{RM}>6.55, \mathrm{PT} \leq 14.75$

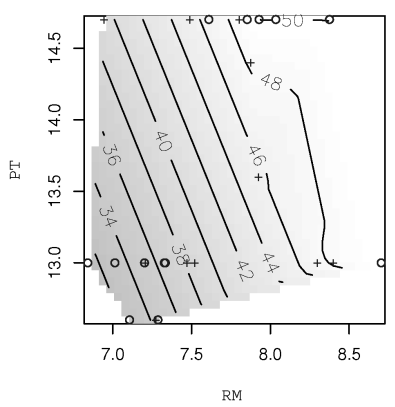

$\mathrm{RM} \leq 6.55, \mathrm{RAD}>16, \mathrm{DIS} \leq 1.42$

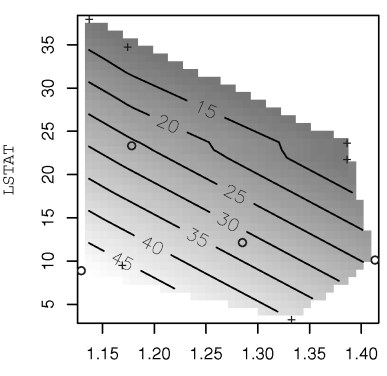

DIS

$\mathrm{RM}>6.55,14.75<\mathrm{PT} \leq 19.45$

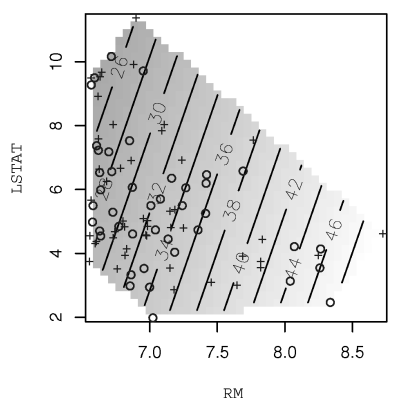

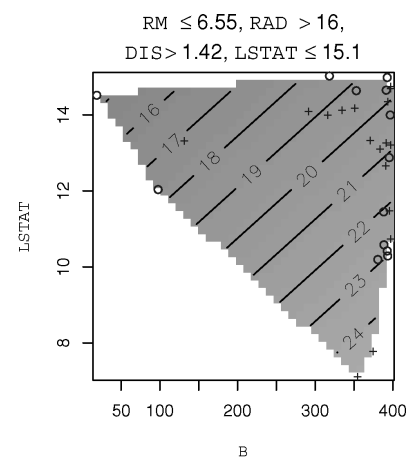

$\mathrm{RM}>6.55, \mathrm{PT}>19.45$

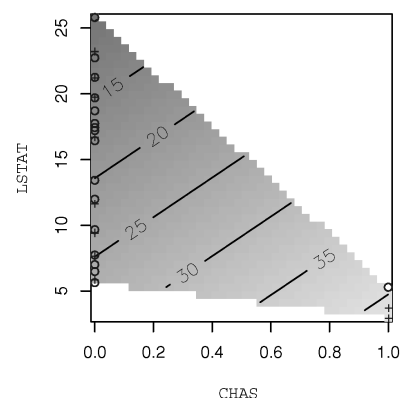

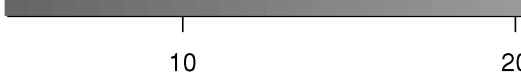

20

30

40

50

MEDV

Fig. 4. Contour plots of the fitted functions in the leaf nodes of the tree in Fig. 3. Data points with positive and negative residuals are marked with + and o symbols, respectively.

two-regressor model to each of the datasets

in $t_{\mathrm{L}}$ and $t_{\mathrm{R}}$.

ii. Compute $S$, the total of the sums of squared residuals in $t_{\mathrm{L}}$ and $t_{\mathrm{R}}$.

Select the smallest value of $x$ that minimizes $S$.

(b) If $X$ is categorical, search for a split of the form

$X \in C$, where $C$ is a subset of the values taken

by $X$. For each $C$ such that $t_{\mathrm{L}}$ and $t_{\mathrm{R}}$ each has at least $n_{0}$ observations, calculate the sample variances of $Z$ in $t_{\mathrm{L}}$ and $t_{\mathrm{R}}$. Select the set $C$ for which the weighted sum of the variances is minimum, with weights proportional to sample sizes.

Step 6. After splitting has stopped, prune the tree using the algorithm described in Breiman et al. (1984, Sec. 8.5) with ten-fold Cross-Validation (CV). Let
Harrison-Rubinfeld

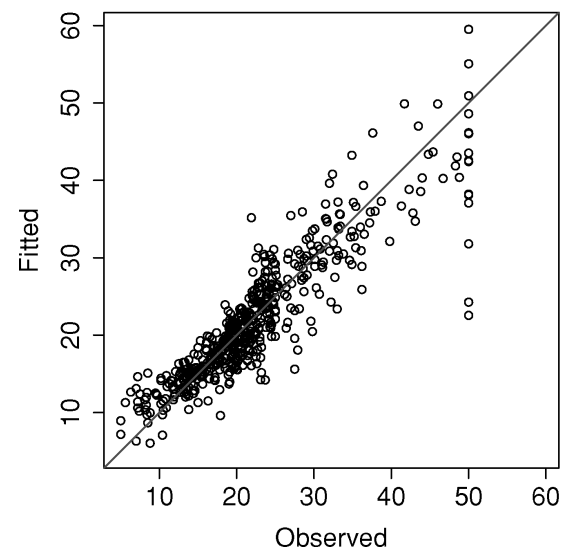

One-regressor tree

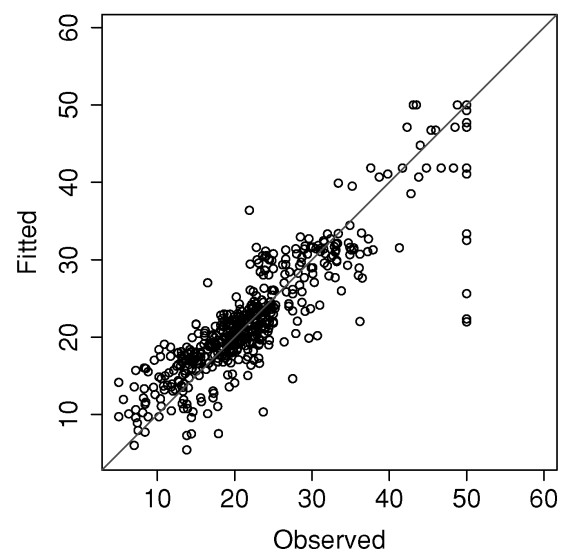

Two-regressor tree

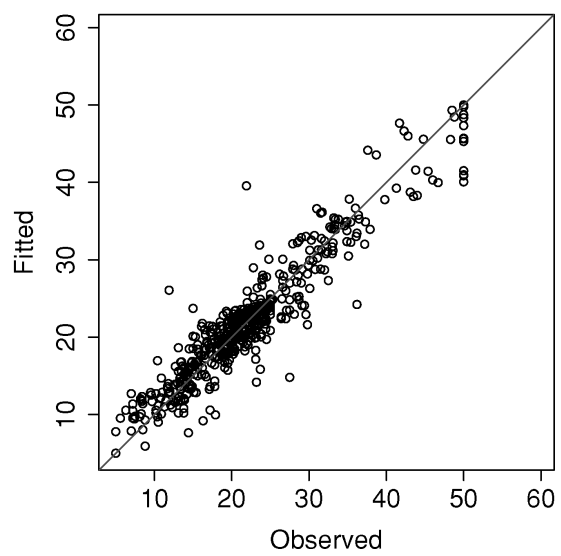

Fig. 5. Fitted versus observed values for the Harrison-Rubinfeld and the piecewise one- and two-regressor models for the Boston data. 
$E_{0}$ be the smallest CV estimate of prediction Mean Square Error (MSE) and let $\alpha$ be a positive number. Select the smallest subtree whose CV estimate of MSE is within $\alpha$ times the standard error of $E_{0}$. We use the default value of $\alpha=0.5$ here and call this the 0.5 -SE rule. To avoid large prediction errors caused by extrapolation, truncate all predicted values so that they lie within the range of the training sample data values in their nodes. The nonparallel contour lines in some of the plots in Fig. 4 are the result of this truncation.

Our split selection approach is uniquely different from that of CART (Breiman et al., 1984) and M5 (Quinlan, 1992), two other regression tree algorithms. CART constructs piecewise constant trees only and it searches for the best variable to split and the best split point simultaneously at each node. This requires the evaluation of all possible splits on every predictor variable. Thus, if there are $K$ quantitative predictor variables each taking $M$ distinct values at a node, $K(M-1)$ splits have to be evaluated. To extend the CART approach to piecewise linear regression, two linear models must be fitted for each candidate split. This means that $2 K(M-1)$ regression models must be computed before a split is found. The corresponding number of regression models for $K$ categorical predictors each having $M$ distinct values is $2 K\left(2^{M-1}-1\right)$. Clearly, the computational cost grows rapidly with $K$ and $M$.

Our approach avoids the computational problem by separating split variable selection from split point selection. To select a variable for splitting, only one regression model is fitted (Step 1 of the algorithm). If the selected variable is quantitative with $M$ distinct values, split set selection requires only $2(M-1)$ models to be fitted (Step 5a). On the other hand, if the selected variable is categorical, no regression fitting is needed to find the set of split values (Step 5b).

M5 uses a hybrid strategy to build a piecewise linear model. First it constructs a large piecewise constant tree using exhaustive search to minimize a weighted sum of standard deviations. Then it prunes the tree using a heuristic argument instead of CV. A single linear model is fitted to each node during pruning.

M5 also treats categorical variables differently. Our piecewise one- and two-regressor models use categorical variables for split selection only; they do not use them as regressors in the linear models. M5, on the other hand, first converts each categorical variable into a vector of zeros and ones and then treats the elements of the vector as quantitative variables for split selection and for regression modeling.

A consequence of these differences in approach is that our method possesses an important property that CART and M5 do not, namely, conditional unbiasedness in split variable selection. We say that a selection method is unbiased if, under the assumption that the predictor variables are statistically independent of the response variable, each predictor has the same chance of being selected. Unbiased- ness is desirable because even a small amount of selection bias in a tree can lead to erroneous or inexplicable conclusions. The reason our method is unbiased can be traced to Step 4 of the algorithm where selection of a variable to split a node is based on contingency table analyses of the residual distribution versus the distributions of the predictor variables. Suppose $X_{1}$ and $X_{2}$ are the regressor variables in a two-regressor model. If the other predictor variables are independent of the response variable, they will also be independent of the residuals. Hence, conditional on $X_{1}$ and $X_{2}$ being the selected regressors, all the other variables have the same chance of being selected to split the node. In contrast, since CART and M5 are based on exhaustive search, their split selection methods are biased toward variables that allow more splits, particularly categorical variables with many distinct values. We demonstrate this with an example in the next section.

\section{Baseball data}

This example utilizes a well-known baseball dataset provided by the American Statistical Association Section on Statistical Graphics for its 1988 data exposition. The data consist of the 1987 opening day salaries and various career and 1986 performance statistics of 263 major league baseball hitters (see Table 3). The purpose of the exposition was to invite statisticians to analyze the data and answer the question, "Are players paid according to their performance?" Fifteen teams took up the challenge and their analyses were published in the conference proceedings.

Table 3. Predictor variables and their definitions for the baseball data

\begin{tabular}{ll} 
Bat86 & \#times at bat, 1986 \\
Hit86 & \#hits, 1986 \\
Hr86 & \#home runs, 1986 \\
Run86 & \#runs, 1986 \\
Rb86 & \#runs batted in, 1986 \\
Wlk86 & \#walks, 1986 \\
Yrs & \#years in major leagues \\
Batcr & \#times at bat, career \\
Hitcr & \#hits, career \\
Hrcr & \#home runs, career \\
Runcr & \#runs, career \\
Rbcr & \#runs batted in, career \\
Wlkcr & \#walks, career \\
Leag86 & League, end 1986 (2 values) \\
Div86 & Division, end 1986 (2 values) \\
Team86 & Team, end 1986 (24 values) \\
Pos86 & Position, 1986 (23 values) \\
Puto86 & \#put outs, 1986 \\
Asst86 & \#assists, 1986 \\
Err86 & \#errors, 1986 \\
Leag87 & League, start 1987 (2 values) \\
Team87 & Team, start 1987 (24 values) \\
\hline
\end{tabular}


Table 4. Hoaglin-Velleman model for $\log ($ Salary)

\begin{tabular}{lrr}
\hline$X$ & $\beta$ & \multicolumn{1}{c}{$t$} \\
\hline Intercept & 3.530 & 31.1 \\
Runcr/Yrs & 0.016 & 9.4 \\
$\sqrt{\operatorname{Run} 86}$ & 0.083 & 4.1 \\
$\min \left[(\text { Yrs }-2)_{+}, 5\right]$ & 0.346 & 22.8 \\
$(\text { Yrs }-7)_{+}$ & -0.041 & -4.4 \\
\hline
\end{tabular}

Hoaglin and Velleman (1995) give a critique of the published results. Defining as "best" the models that are "most parsimonious, most interpretable, and best fitting", they conclude that a log transformation of Salary is most successful, and that the best predictor variables are Yrs, Runcr/Yrs, and a 1986 performance measure. They also find seven outliers in the data. Six are due to errors in the data and are omitted from their analysis. The seventh outlier (Pete Rose) is retained because it is not erroneous. The model fitted to the 257 cases is given in Table 4 (Hoaglin and Velleman use base-10 log whereas we use natural log here).

Although the $t$-statistics in Table 4 are all highly significant, they again do not have their usual statistical meaning because the dataset is essentially the whole population of major league hitters for 1987. Besides, even if the data were a random sample, the $t$-statistics are expected to be inflated by the process of variable selection and transformation. Despite this, the variables in the model make sense: Yrs accounts for experience, Runcr/Yrs for productivity rate, and Run86 for 1986 performance. What is difficult to explain is the negative coefficient for $(\mathrm{Yrs}-7)_{+}$. It appears to suggests that the players were penalized for experience beyond 7 years.

Using the data from all 263 hitters, but with the errors replaced by the correct values listed in Hoaglin and Velleman (1995), our piecewise one-regressor model tree has three leaf nodes. It splits twice on Yrs, as shown on the left side of Fig. 6. If Yrs $\leq 3$, the best predictor is Runcr; if $3<\operatorname{Yrs} \leq 6$, the best predictor is Hitcr; otherwise the best predictor is Hit86. Our model is thus quite similar to that of Hoaglin and Velleman. While the latter is a three-piece model divided along Yrs at two and seven, ours divides Yrs at three and six. Our model similarly suggests that salary depends on career performance for Yrs $\leq 6$; beyond 6 years,

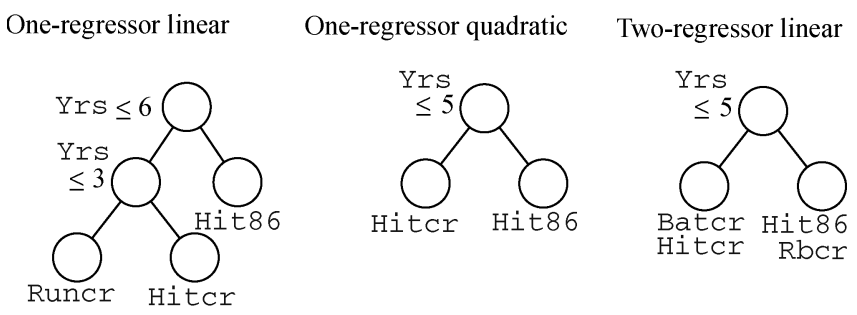

Fig. 6. Three regression tree models for the baseball data.
1986 performance is more important. Figure 7 shows the data and fitted functions in the three leaf nodes of the tree.

The split of Yrs into three intervals suggests a curvature effect. We can better model this with a piecewise oneregressor quadratic model. This tree is shown in the middle of Fig. 6. It is simpler, with only one split-again on Yrs. The best predictor variable is Hitcr if Yrs $\leq 5$ and Hit86 otherwise. Again, salary tends to increase with career performance for the junior players and with 1986 performance for the senior players. Plots of the fitted curves are shown in Fig. 8.

The tree for our piecewise two-regressor linear model is displayed on the right side of Fig. 6. It has the same structure as that for the one-regressor quadratic model, except that two variables are selected as linear regressors in each leaf node. We observe from the contour plots of the fitted functions in Fig. 9 that Batcr and Hitcr are the most important predictor variables for players with five or fewer years of experience. As may be expected, the plot shows that these two variables are highly correlated. Furthermore, except for a couple of players, most of the junior ones have lower salaries. For players with more than 5 years of experience, the most important predictors are Hit86 and Rbcr. It is noteworthy that the sample correlation between these two variables here is quite low at 0.18 . Many of the highestpaid players belong to this group. Figure 10 shows that the fit of this model is as good as that of the Hoaglin-Velleman model.

Figure 11 shows the CART and M5 models for these data. The M5 tree is obtained using the WEKA (Witten and Frank, 2000) implementation. The similarity in the splits is due to CART and M5 both constructing piecewise constant regression trees prior to pruning. CART minimizes the residual sum of squares but M5 minimizes a weighted sum of standard deviations. This is the reason for the different split values at the root node.

Given the prominent role of Yrs in our models and that of Hoaglin and Velleman, it may seem odd that this variable is completely absent from the CART tree. (Yrs is also not used to split the M5 tree, but it is used as linear predictor in the leaf node models.) CART and M5 split first on Batcr instead. Since the latter is positively correlated with Yrs, we conjecture that Batcr is acting as a surrogate to Yrs. The reason it is chosen is most likely due to selection bias - Batcr has 256 permissible splits while Yrs has only 20. Another possible manifestation of the bias appears in the CART split on the categorical variable Team86, which neither the Hoaglin-Velleman nor our tree models find important. It turns out that Team86 has 22 distinct categories at that node. Therefore it yields $2^{21}-1=2097151$ permissible splits. As a result, the residual sum of squares can be reduced much more by splitting on Team 86 than on an ordered quantitative variable. M5 is similarly affected by this selection bias. It uses Team86 and Team87 as split variables in the lower branches of the tree, but these branches are removed by pruning. Nonetheless, some tell-tale evidence 

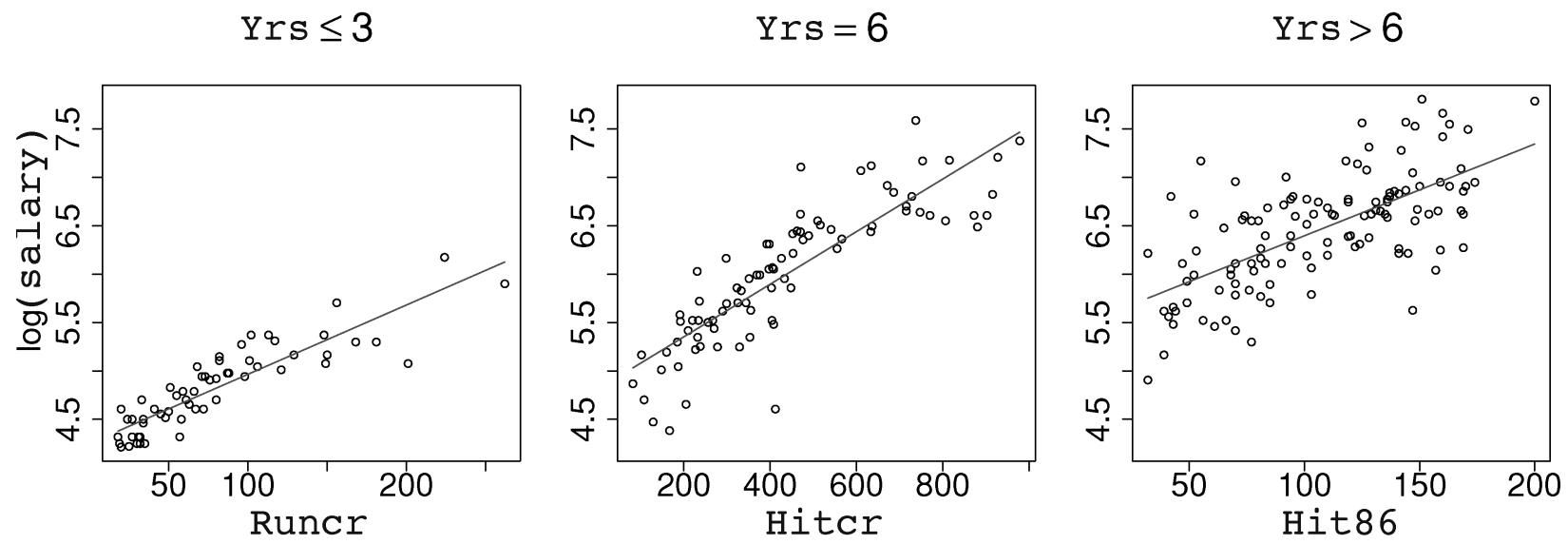

Fig. 7. Fitted functions and data for the one-regressor linear tree model for the baseball data.

involving these variables can be found in the piecewise linear regression functions $L_{1}-L_{5}$ in the leaf nodes. For example:

$$
\begin{aligned}
& 10^{4} L_{1} \\
& =41300+97.2 \text { Yrs }-29.2 \text { Bat86 + 18.8 Batcr } \\
& +112 \mathrm{Hit} 86-44.7 \mathrm{Hitcr}+17.1 \mathrm{Wlk} 86+2.2 \mathrm{Hrcr} \\
& +0.456 \text { Runcr }+35.6 \mathrm{Rbcr}-1.68 \mathrm{Wlkcr} \\
& \text { + } 63 \text { I (Team86 } \in \text { Atl, Bal, Bos, Cal, Chi, Cin, Cle, } \\
& \text { Det, Hou, LA, NY, Oak, Tor }\})+418 I(\text { Team86 = StL }) \\
& \text { +875I(Team87 } \in \text { \{Atl, Bal, Bos, Chi, LA, NY, Oak, } \\
& \text { StL, Tor }\})+534 I(\text { Team8 } \in\{\text { Cal, Cin, Cle, Det, } \\
& \text { Hou, KC, Mil, Min, Mon, Phi, SD, SF, Tex\}). }
\end{aligned}
$$

Notice the counter-intuitive change in signs between the coefficients of Bat86 and Batcr and between those of Hit86 and Hitcr. Since the M5 tree contains five linear regression functions, it is five times as hard to interpret as a traditional multiple linear regression model.

\section{Prediction accuracy}

We now put aside the issue of model interpretation and consider how our methods compare against other methods
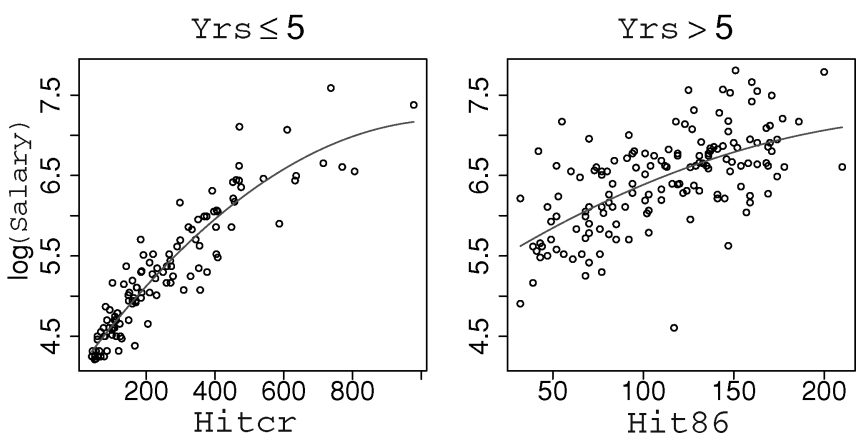

Fig. 8. Fitted functions and data for the one-regressor quadratic tree model for the baseball data. in terms of prediction accuracy when applied to real data. Since there are few published empirical studies comparing statistical with machine learning regression methods, we include some of the most well-known algorithms from each discipline. The results reported here are obtained using 27 algorithms and 52 datasets.

\subsection{Datasets}

The datasets are listed in Table 5 together with information on sample sizes, numbers of quantitative and categorical predictor variables, and their sources. Sample size ranges from 96 to 21252 , number of quantitative predictor variables from one to 28 , and number of categorical variables from zero to six. All binary predictor variables are treated as quantitative. Figure 12 summarizes the information in a graph. The datasets mostly come from books and journal articles, although several are from Statlib (http://lib.stat.cmu.edu/) and the UCI data repository (Blake and Merz, 1998). Observations with missing or incorrect values are removed.

For the six datasets accompanied by test sets, we apply each algorithm to the training set and compute the prediction MSE of the fitted model on the test set. For the other datasets, we use ten-fold CV to estimate the prediction MSE. That is, we first randomly divide each dataset into ten roughly equal-sized subsets. Then we set aside one subset in turn, pool the observations in the other nine subsets, apply each algorithm to the combined data, and compute the prediction MSE of the fitted model on the set-aside subset. The average of the ten results yields the CV estimate.

\subsection{Algorithms}

Table 6 lists the 27 algorithms. Unless stated otherwise, each algorithm is used with its default parameter values. For those algorithms that cannot directly deal with categorical predictor variables, we follow the standard practice 

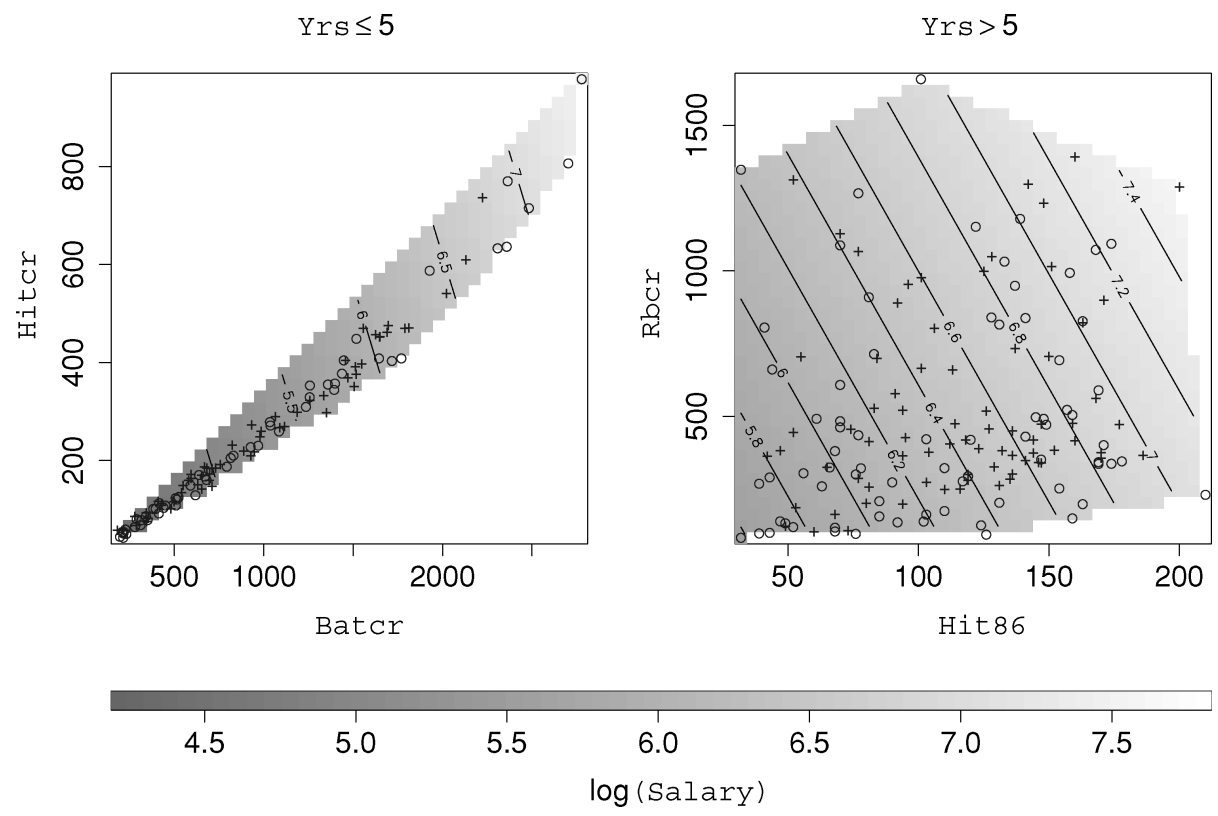

Fig. 9. Data and contour plots of the piecewise two-regressor model for the baseball data. Points associated with positive and negative residuals are denoted by + and $o$, respectively.
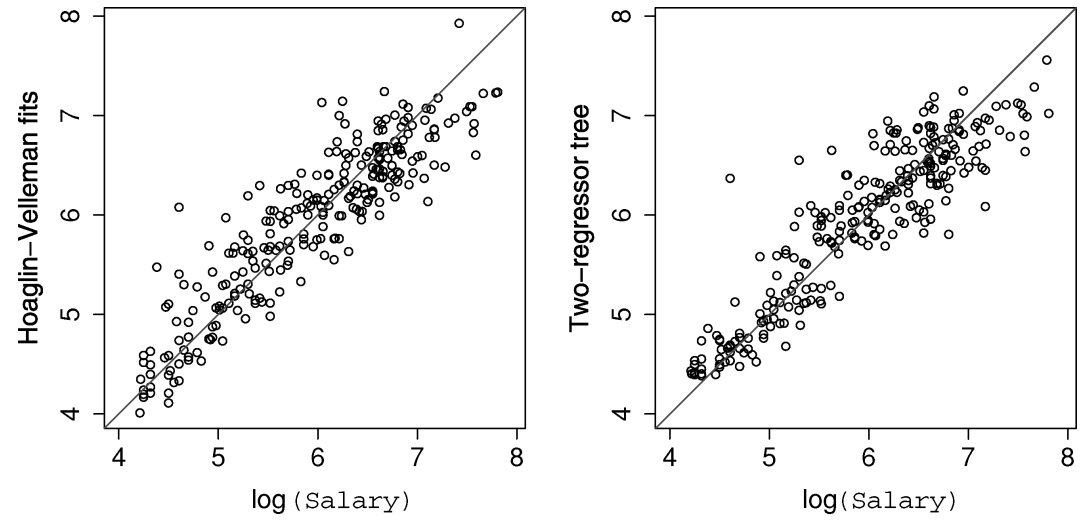

Fig. 10. Fitted versus observed $\log ($ Salary) for the baseball data.

CART

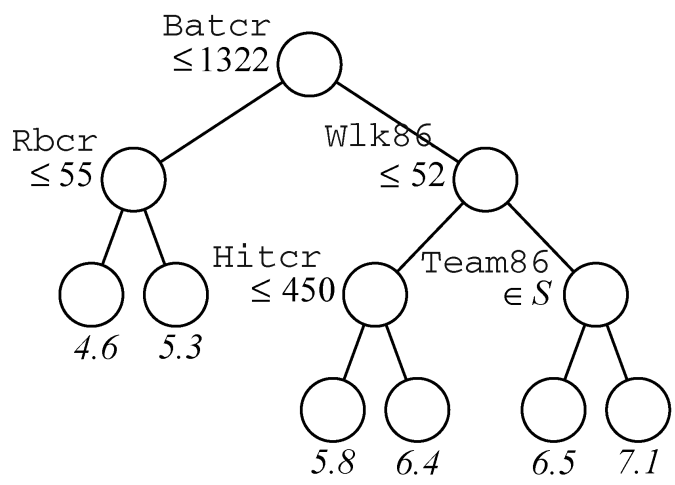

M5

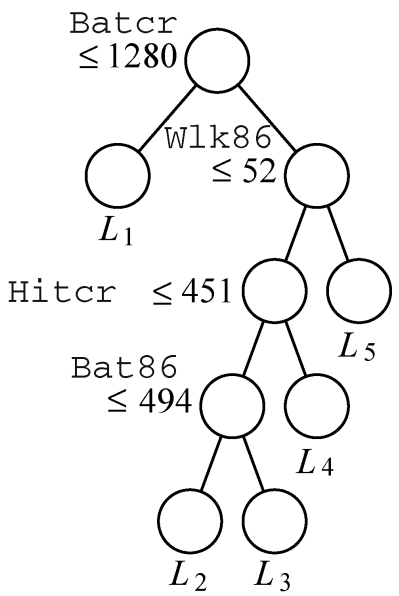

Fig. 11. CART piecewise constant and M5 piecewise multiple linear models for the baseball data. The fitted values for the CART model are given in italics beneath the leaf nodes. $S$ consists of the teams: Atlanta, California, Cincinnati, Cleveland, Detroit, Houston, Los Angeles, Oakland, Pittsburgh, San Diego, San Francisco, and Seattle. $L_{1}-L_{5}$ are linear functions. 
Table 5. Datasets ( $N$ denotes the number of training cases, $N^{*}$ the number of test cases (if any), $Q$ the number of quantitative predictors, and $C$ the number of categorical predictors (category sizes in parentheses))

\begin{tabular}{|c|c|c|c|c|c|}
\hline Name & $N$ & $N^{*}$ & $Q$ & $C$ & Source \\
\hline Abalone & 4177 & & 7 & $1(3)$ & UCI \\
\hline Ais & 202 & & 11 & $1(9)$ & Cook and Weisberg (1994) \\
\hline Alcohol & 2467 & & 12 & $6(3,3,3,4,4,6)$ & Kenkel and Terza (2001) \\
\hline Amenity & 3044 & & 19 & $2(3,4)$ & Chattopadhyay (2003) \\
\hline Attend & 838 & & 7 & $2(3,29)$ & Cochran (2002) \\
\hline Baseball & 263 & & 18 & $2(23,24)$ & Statlib \\
\hline Baskball & 96 & & 4 & 0 & Simonoff (1996) \\
\hline Boston & 506 & & 13 & 0 & Belsley et al. (1980) \\
\hline Boston2 & 506 & & 13 & $1(92)$ & Belsley et al. (1980) \\
\hline Budget & 1729 & & 10 & 0 & Bollino et al. (2000) \\
\hline Cane & 3775 & & 6 & $3(14,15,24)$ & Denman and Gregory (1998) \\
\hline Cardio & 375 & & 6 & $3(7,8,12)$ & Bryant and Smith (1996) \\
\hline College & 694 & & 23 & $1(3)$ & Statlib \\
\hline County & 3114 & & 12 & $1(46)$ & Harrell (2001) \\
\hline Cps & 534 & & 7 & $3(3,3,6)$ & Berndt (1991) \\
\hline Cps95 & 21252 & 42504 & 8 & $6(2,3,4,5,7,9)$ & ftp.stat.berkeley.edu/pub/datasets/fam95.zip \\
\hline $\mathrm{Cpu}$ & 209 & & 6 & $1(30)$ & UCI \\
\hline Deer & 654 & & 10 & $3(2,6,7)$ & Onoyama et al. (1998) \\
\hline Diabetes & 375 & & 14 & $1(3)$ & Harrell (2001) \\
\hline Diamond & 308 & & 1 & $3(3,5,6)$ & Chu (2001) \\
\hline Edu & 1400 & & 5 & 0 & Martins (2001) \\
\hline Engel & 11986 & 11986 & 5 & 0 & Delgado and Mora (1998) \\
\hline Enroll & 258 & & 6 & 0 & Liu and Stengos (1999) \\
\hline Fame & 1318 & & 21 & $1(7)$ & Cochran $(2000)$ \\
\hline Fat & 252 & & 14 & 0 & Penrose et al. (1985) \\
\hline Fishery & 6806 & & 11 & $3(3,5,6)$ & Fernandez et al. (2002) \\
\hline Hatco & 100 & & 12 & $1(3)$ & Hair et al. (1998) \\
\hline Houses & 6880 & 13760 & 8 & 0 & Pace and Barry (1997) \\
\hline Insur & 2182 & & 4 & $2(7,9)$ & Hallin and Ingenbleek (1983) \\
\hline Labor & 2953 & & 18 & 0 & Aaberge et al. (1999) \\
\hline Labor2 & 5443 & 5443 & 17 & 0 & Laroque and Salanie (2002) \\
\hline Laheart & 200 & & 13 & $3(4,4,5)$ & Afifi and Azen (1979) \\
\hline Medicare & 4406 & & 21 & 0 & Deb and Trivedi (1997) \\
\hline Mpg & 392 & & 6 & $1(3)$ & UCI \\
\hline Mpg2001 & 852 & & 5 & $5(3,3,5,12,42)$ & www.fueleconomy.gov \\
\hline Mumps & 1523 & & 3 & 0 & Statlib \\
\hline Mussels & 201 & & 3 & $1(5)$ & Cook (1998) \\
\hline Ozone & 330 & & 8 & 0 & Breiman and Friedman (1988) \\
\hline Pole & 5000 & 10000 & 26 & 0 & Weiss and Indurkhya (1995) \\
\hline Price & 159 & & 15 & 0 & UCI \\
\hline Rate & 144 & & 9 & 0 & Lutkepohl et al. (1999) \\
\hline Rice & 171 & & 13 & $2(3,3)$ & Horrace and Schmidt (2000) \\
\hline Scenic & 113 & & 9 & $1(4)$ & Neter et al. (1996) \\
\hline Servo & 167 & & 2 & $2(5,5)$ & UCI \\
\hline Smsa & 141 & & 9 & $1(4)$ & Neter et al. (1996) \\
\hline Spouse & 11136 & 11136 & 21 & 0 & Olson (1998) \\
\hline Strike & 625 & & 4 & $1(18)$ & Statlib \\
\hline $\mathrm{Ta}$ & 324 & & 3 & $3(3,30,40)$ & Authors \\
\hline Tecator & 215 & & 10 & 0 & Statlib \\
\hline Tree & 100 & & 8 & 0 & Rawlings (1988) \\
\hline Triazine & 186 & & 28 & 0 & Torgo (1999) \\
\hline Wage & 3380 & & 13 & 0 & Schafgans (1998) \\
\hline
\end{tabular}




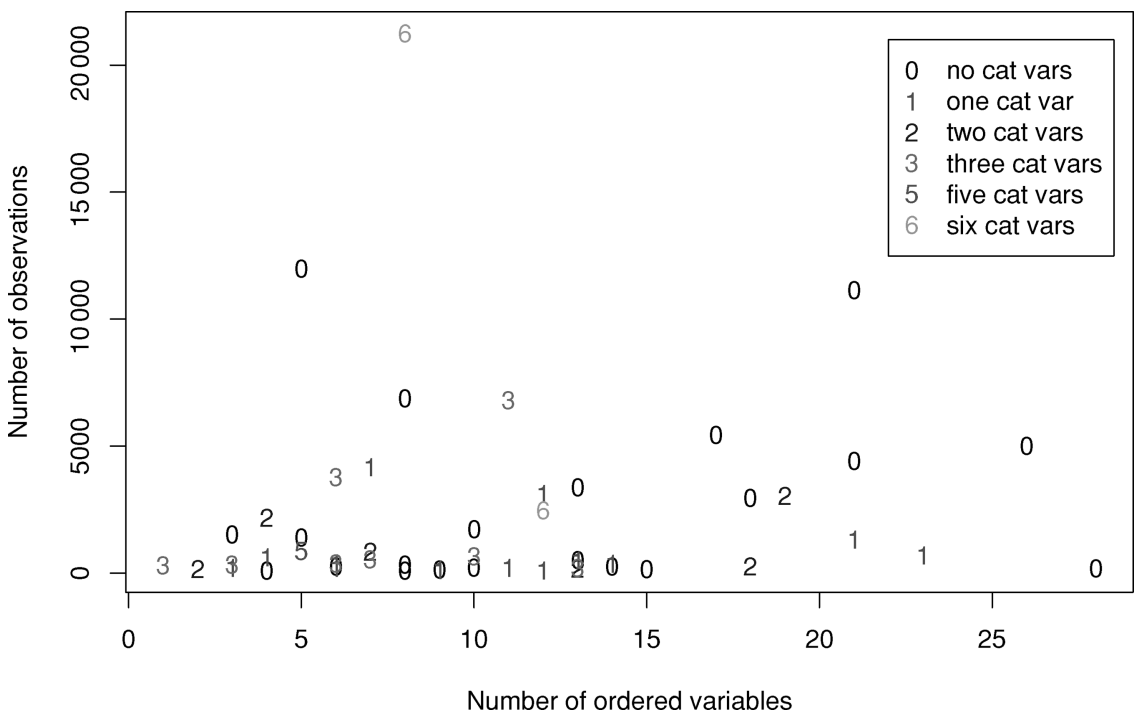

Fig. 12. Sample size versus number of predictor variables for the 52 datasets. Plot symbol indicates the number of categorical variables.

of converting them to zero-one dummy vectors. Each vector component is then treated as a quantitative predictor variable.

CART. Piecewise constant regression tree (Breiman et al., 1984). CART is a registered trademark of California Statistical Software, Inc. We use version 4 of the Windows

Table 6. The 27 algorithms considered in this study

\begin{tabular}{ll}
\hline Cart & CART piecewise constant \\
$\mathrm{Cr}$ & CUBIST rule-based model \\
$\mathrm{Ci}$ & Cr and nearest-neighbor (composite) \\
$\mathrm{Crb}$ & Boosted Cr (committee model) \\
gam & Generalized additive model \\
$\mathrm{Gc}$ & GUIDE piecewise constant \\
$\mathrm{Gl}$ & GUIDE piecewise simple linear \\
$\mathrm{Gq}$ & GUIDE piecewise simple quadratic \\
$\mathrm{Gm}$ & GUIDE piecewise multiple linear \\
$\mathrm{Gs}$ & GUIDE piecewise stepwise linear \\
$\mathrm{Gs} 2$ & GUIDE two-regressor stepwise linear \\
$\mathrm{Gf} 2$ & GUIDE two-regressor forward linear \\
$\mathrm{lad}$ & Least absolute deviations regression \\
$\mathrm{lr}$ & Least squares linear regression \\
$\mathrm{mars}$ & MARS \\
$\mathrm{mart}$ & MART \\
$\mathrm{Mc}$ & M5 piecewise constant \\
$\mathrm{Mcb}$ & Bagged Mc \\
$\mathrm{Mm}$ & M5 piecewise multiple linear \\
$\mathrm{Mmb}$ & Bagged Mm \\
$\mathrm{nnet}$ & Neural network \\
$\mathrm{pol}$ & POLYMARS \\
$\mathrm{ppr}$ & Projection pursuit regression \\
$\mathrm{Rc}$ & RT piecewise constant \\
$\mathrm{Rm}$ & RT piecewise multiple linear \\
$\mathrm{Rp}$ & RT piecewise partial linear \\
$\mathrm{rreg}$ & Huber's robust regression \\
\hline &
\end{tabular}

implementation (Steinberg and Colla, 1995), with ten-fold $\mathrm{CV}$ and the default 0-SE rule. The minimal node size is ten except for the Cps95 dataset where the value is changed to 100 because of the program's memory limitations.

CUBIST. A rule-based algorithm due to R. Quinlan (www.rulequest.com/cubist-info.html). We use Release 1.10. Three type of models are studied: rule-based only $(\mathrm{Cr})$, composite $(\mathrm{Ci})$, and committee $(\mathrm{Crb})$ with five members. The $\mathrm{Ci}$ model combines $\mathrm{Cr}$ with a nearest-neighbor method. $\mathrm{Crb}$ is a boosted version of $\mathrm{Cr}$.

GAM. Generalized additive model (Hastie and Tibshirani, 1990). We use the S-Plus function gam with the Gaussian family and nonparametric smoothing splines (option s).

GUIDE. Generalized regression tree (Loh, 2002). Gc and Gm denote piecewise constant and piecewise multiple linear models. Categorical variables are used for splitting and for regression modeling (via dummy vectors) in Gm. Our proposed piecewise simple linear and simple quadratic models are denoted by Gl and Gq, respectively. Gs denotes the method where forward and backward stepwise regression is used in each node. If the number of regressors is limited to two, the method is denoted by Gs2. Finally, Gf 2 denotes the method using two-regressor forward-only stepwise regression at each node. The trees are pruned with the default 0.5-SE rule.

Least absolute deviations regression. We use the S-Plus function l1fit.

Least-squares linear regression. We use the $\mathrm{R}$ function $\mathrm{lm}$.

M5. Piecewise constant and linear regression tree. We use the implementation in version 3.2 of WEKA (Witten and Frank, 2000). Mc denotes piecewise constant and Mm piecewise multiple linear. If bagging is employed, we use ten 
Table 7. Geometric means of RMSE relative to linear regression, in increasing order (algorithms in the first row are not significantly different from $\mathrm{Crb}$ )

\begin{tabular}{ccccccccccccc}
\hline Crb & Mmb & Mm & Ci & \multirow{2}{*}{ r } & Gs & Gm & gam & Gf2 & Gs2 & mars & Mcb & ppr \\
0.78 & 0.80 & 0.82 & 0.82 & 0.84 & 0.86 & 0.86 & 0.90 & 0.90 & 0.90 & 0.91 & 0.91 & 0.92 \\
nnet & mart & Gl & Rp & Cart & Rm & Gq & Mc & rreg & Gc & Rc & lad & pol \\
0.94 & 0.96 & 0.96 & 0.97 & 0.97 & 0.98 & 0.98 & 0.99 & 1.0 & 1.0 & 1.0 & 1.1 & 1.2 \\
\hline
\end{tabular}

iterations. The resulting methods are denoted by Mcb and Mmb, respectively.

MARS. Multivariate adaptive regression splines (Friedman, 1991). We use the $\mathrm{R}$ function mars in the mda library with parameter values degree $=1$, penalty $=2$, and thresh $=0.001$.

MART. Multivariate adaptive regression tree (Friedman, 2001). This is a stochastic gradient boosting algorithm applied to regression trees. We use the software from wwwstat.stanford.edu/jhf/mart.html with 10-fold CV and 200 boosting iterations.

NNET. Neural network using the $\mathrm{R}$ function nnet with size $=3$, decay $=0.001$, linout $=$ TRUE, skip $=$ TRUE, and maxit $=200$.

POLYMARS. An adaptive regression procedure using piecewise linear splines (Kooperberg et al., 1997). We use the $\mathrm{R}$ function polymars in the polspline library. The gcv option is used for model selection. The maximum number of basis functions is $\min \left(6 n^{1 / 3}, n / 4,100\right)$ and the maximum number of knots per predictor is $\min (20$, round $(n / 4))$, where $n$ is the sample size.

Projection pursuit regression. (Friedman and Stuetzle, 1981). We use the $R$ function $p p r$ with optlevel $=2$ in the modreg library.

Robust regression with M-estimate. (Huber, 1981, p. 194). We use the $R$ function $r l m$ with init $=1 \mathrm{~s}, \mathrm{k} 2=1.345$, maxit $=20$, and acc $=1 e-4$ in the MASS library (Venables and Ripley, 1999).

RT. A regression tree algorithm due to Torgo (1999). Like M5, it first grows a piecewise constant tree and then fits various linear models to the leaf nodes during pruning. We use version 4 of the software. Rc denotes piecewise constant, $\mathrm{Rm}$ piecewise multiple linear, and $\mathrm{Rp}$ piecewise partial linear with a bandwidth size of ten.

\subsection{Results}

Because the measurement scale of the response variable varies from one dataset to another, it is necessary to standardize the prediction MSEs. Let the square root of the prediction MSE be denoted by RMSE. We divide the RMSE of each algorithm by that of least squares linear regression (lr) and then take its logarithm. We call the result the $\log$ relative root mean squared error (LRMSE). A positive
LRMSE indicates that the algorithm is less accurate than Ir.

Table 7 gives the geometric means of the RMSE relative to linear regression and Fig. 13 displays them in a barplot. The two algorithms with the lowest geometric means are the ensemble methods $\mathrm{Crb}$ and Mmb, which have geometric means of 0.78 and 0.80 , respectively. They are followed by Mm, Ci, Cr (all due to Quinlan), Gs, Gm, and gam. After these come our proposed two-regressor tree methods Gf2 and Gs2, which employ forward-only and forward-backward stepwise regression, respectively.

It would be erroneous to conclude that if one algorithm has a smaller geometric mean RMSE than another, then the former always has smaller prediction error than the latter, because there is substantial variability within each algorithm across datasets. This can be seen in Fig. 14, which shows boxplots of the relative RMSE values by algorithm, ordered by their medians. We see there are datasets for which some algorithms (e.g., Crb and Gc) have RMSE values as low as one-tenth that of linear regression. On the other hand, there are also datasets for which algorithms such as mart and Mcb have relative RMSEs that are two or more.

To find out whether the differences in RMSEs are statistically significant, we fit a mixed-effects model to the LRMSE values, using algorithm as a fixed effect, dataset as a random effect, and their interaction as another independent random effect in place of the usual "error term". Calculations based on Tukey $95 \%$ simultaneous confidence intervals for pairwise differences show that algorithms with geometric mean RMSEs less than 0.923 are not significantly different from $\mathrm{Crb}$. Thus differences among the prediction RMSEs of the top 13 algorithms (listed in the first row of Table 7) are not statistically significant. Our piecewise two-regressor trees Gf2 and Gs2 belong to this group but not Gl and Gq. Also belonging to this group are gam, mars, Mcb, and ppr. Within this top group of 13, only Gf 2 and Gs2 yield interpretable and visualizable models.

Although regression trees are often thought to be more interpretable than other methods, it should not be forgotten that interpretability depends on the complexity of a tree. All other things being equal, a tree with many leaf nodes takes more effort to interpret than one with few nodes. Figure 15 shows how the 13 regression tree algorithms compare in terms of mean number of leaf nodes. The piecewise constant tree methods (Cart, Gc, Rtc, and Mc) tend to produce trees with many leaf nodes - 20 for Gc, 34 for all versions of Rt, and 55 for Cart. This makes them quite hard to interpret 


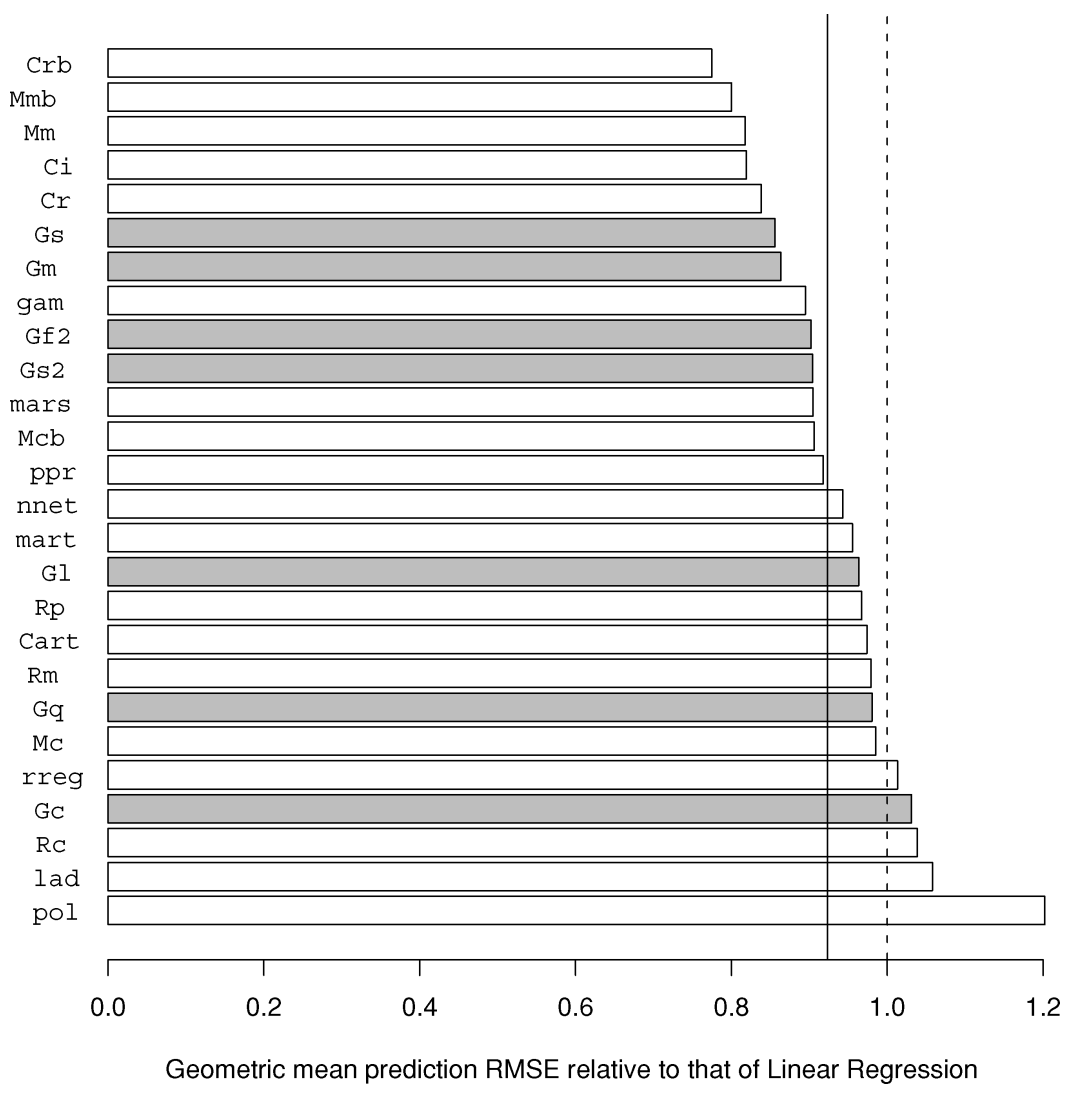

Fig. 13. Barplot of geometric mean prediction RMSE relative to that of linear regression. The RMSE of an algorithm is not statistically significantly different from that of $\mathrm{Crb}$ if its associated bar ends to the left of the solid vertical line. The GUIDE algorithms are in gray.

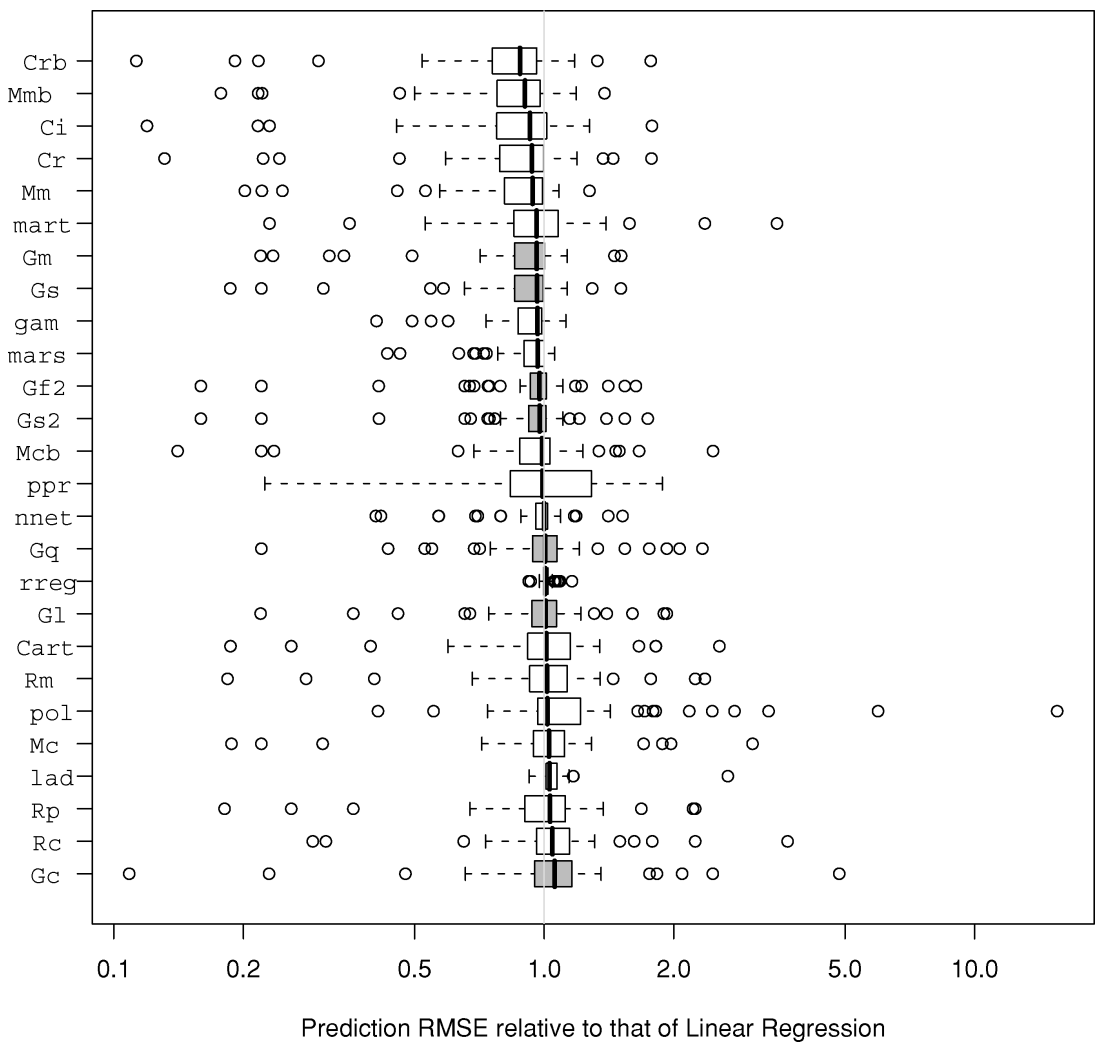

Fig. 14. Boxplots of RMSE relative to that of linear regression, ordered by their medians. GUIDE algorithms are in gray. 


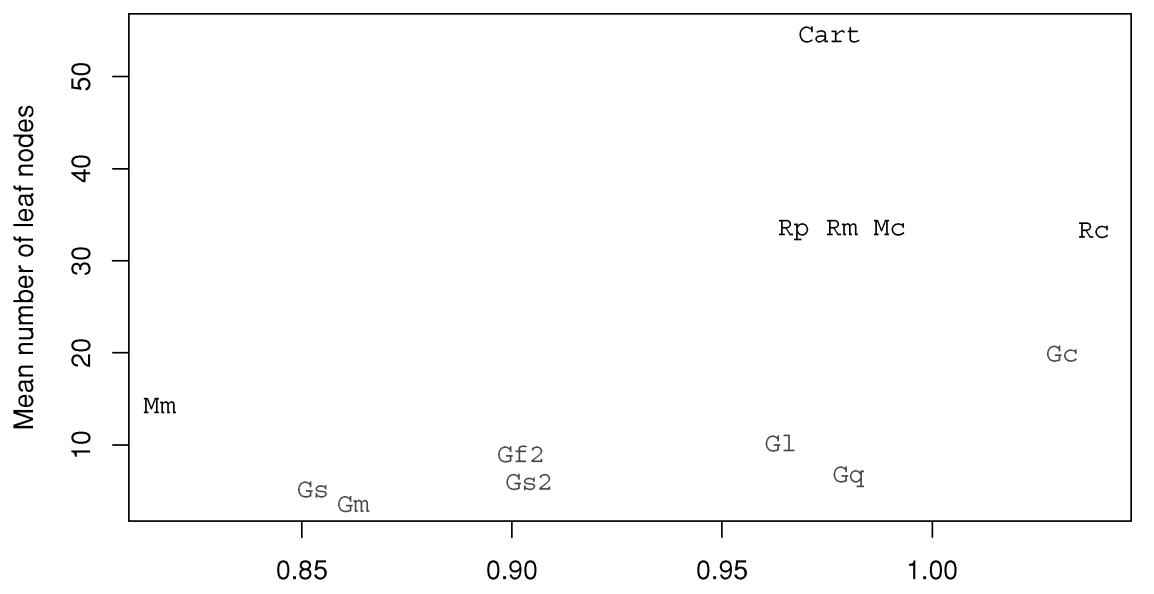

Geometric mean prediction RMSE relative to that of linear regression

Fig. 15. Mean number of leaf nodes versus geometric mean of prediction RMSE relative to that of linear regression. The plot symbols for Gf2 and Gs2 almost coincide.

in practice. Gm has the lowest average of 3.7. But its trees are not necessarily interpretable because each node is fitted with a multiple linear model. The class of tree methods that lie in between, namely the piecewise one- and two-regressor models, strikes a compromise with relatively simple node models and relatively compact trees: Gl, Gq, Gf2, and Gs2 have on average $10.3,6.7,6.1$, and 6.1 leaf nodes, respectively.

\section{Asymptotic behavior of regression estimates}

We state and prove here an asymptotic consistency theorem for piecewise two-regressor models. The theorem provides hope that the good empirical performance of the method will scale up to arbitrarily large sample sizes. First, we establish some notation. Let $\left(Y_{1}, \mathbf{X}_{1}\right),\left(Y_{2}, \mathbf{X}_{2}\right), \ldots,\left(Y_{n}, \mathbf{X}_{n}\right)$ be $n$ independent observations forming the training sample. The $Y$ values are real valued and the $\mathbf{X}$ values take values in a $d$-dimensional compact hyper-rectangle $C$ in the Euclidean space $R^{d}$. Suppose that $T_{n}$ is a partition of $C$ consisting of sets that are also hyper-rectangles in $R^{d}$. For any $t \in T_{n}$, we will denote by $\delta(t)$ the diameter of $t$ defined as $\delta(t)=\sup \{\|\mathbf{x}-\mathbf{z}\|: \mathbf{x}, \mathbf{z} \in t\}$, where $\|$.$\| is the usual Eu-$ clidean norm of a vector, and we define the norm of the partition $T_{n}$ as $\left|T_{n}\right|=\sup \left\{\delta(t): t \in T_{n}\right\}$.

For $t \in T_{n}$, let $N_{t}$ be the number of $\mathbf{X s}$ in $t, N_{n}=$ $\min \left\{N_{t}: t \in T_{n}\right\}$, and $\overline{\mathbf{X}}_{t}=N_{t}^{-1} \sum_{\mathbf{X}_{i} \in t} \mathbf{X}_{i}$. Given a nonnegative integer $m$, let $\mathbf{U}$ be a set of pairs $\mathbf{u}=\left(u_{1}, u_{2}\right)$ of non-negative integers such that $[\mathbf{u}] \leq m$, where $[\mathbf{u}]=u_{1}+$ $u_{2}$. Let $s(\mathbf{U})$ denote the size of $\mathbf{U}$. For $\mathbf{x}, \mathbf{z} \in t$, define the $s(\mathbf{U})$-dimensional vector $\Gamma(\mathbf{x}, \mathbf{z} ; p, q)=\left[\{\delta(t)\}^{-[\mathbf{u}]}\left(x_{p}-\right.\right.$ $\left.\left.z_{p}\right)^{u_{1}}\left(x_{q}-z_{q}\right)^{u_{2}}\right]_{\mathbf{u} \in \mathbf{U}}$, where $1 \leq p, q \leq d, \mathbf{x}=\left(x_{1}, x_{2}, \ldots\right.$, $\left.x_{d}\right)$, and $\mathbf{z}=\left(z_{1}, z_{2}, \ldots, z_{d}\right)$. Also define $D(p, q, t)=$ $\sum_{\mathbf{X}_{i} \in t} \Gamma^{T}\left(\mathbf{X}_{i}, \overline{\mathbf{X}}_{t} ; p, q\right) \Gamma\left(\mathbf{X}_{i}, \overline{\mathbf{X}}_{t} ; p, q\right)$.

Consider the least-squares fit of a two-regressor polynomial model of order $m$ in partition $t$ and let $p_{t}$ and $q_{t}$ be the indices of the two selected variables. Then the estimate of the regression function $g(\mathbf{x})=E(Y \mid \mathbf{X}=\mathbf{x})$ for $\mathbf{x} \in t$ is given by the expression:

$\hat{g}(\mathbf{x})=\Gamma\left(\mathbf{x}, \overline{\mathbf{X}}_{t} ; p_{t}, q_{t}\right) D^{-1}\left(p_{t}, q_{t}, t\right) \sum_{\mathbf{X}_{i} \in t} \Gamma^{T}\left(\mathbf{X}_{i}, \overline{\mathbf{X}}_{t} ; p_{t}, q_{t}\right) Y_{i}$.

Let $\lambda_{t}$ denote the smallest eigenvalue of $D\left(p_{t}, q_{t}, t\right)$ and write $\lambda_{n}=\min \left\{\lambda_{t}: t \in T_{n}\right\}$. Furthermore, let $\psi(a \mid \mathbf{x})=$ $E[\exp \{a|Y-g(\mathbf{x})|\} \mid \mathbf{X}=\mathbf{x}]$ for any $a>0$ such that the expectation is finite.

Theorem 1. Assume that the regression function is continuous in C. Suppose that $\left|T_{n}\right|$ and $\log n / N_{n}$ tend to zero and that $\lambda_{n}$ remains bounded away from zero in probability as $n \rightarrow \infty$. If there exists $a>0$ such that $\psi(a \mid \mathbf{x})$ is bounded in $C$, then $\sup \{|\hat{g}(\mathbf{x})-g(\mathbf{x})|: \mathbf{x} \in C\} \rightarrow 0$ in probability as $n \rightarrow \infty$.

Proof. First observe that

$$
\begin{aligned}
\hat{g}(\mathbf{x})= & \Gamma\left(\mathbf{x}, \overline{\mathbf{X}}_{t} ; p_{t}, q_{t}\right) D^{-1}\left(p_{t}, q_{t}, t\right) \sum_{\mathbf{X}_{i} \in t} \Gamma^{T} \\
& \times\left(\mathbf{X}_{i}, \overline{\mathbf{X}}_{t} ; p_{t}, q_{t}\right) g\left(\mathbf{X}_{i}\right)+\Gamma\left(\mathbf{x}, \overline{\mathbf{X}}_{t} ; p_{t}, q_{t}\right) D^{-1} \\
& \times\left(p_{t}, q_{t}, t\right) \sum_{\mathbf{X}_{i} \in t} \Gamma^{T}\left(\mathbf{X}_{i}, \overline{\mathbf{X}}_{t} ; p_{t}, q_{t}\right) \epsilon_{i},
\end{aligned}
$$

where $\epsilon_{i}=Y_{i}-g\left(\mathbf{X}_{i}\right)$. Replacing $g\left(\mathbf{X}_{i}\right)$ with $g(\mathbf{x})+$ $\left\{g\left(\mathbf{X}_{i}\right)-g(\mathbf{x})\right\}$ in the first term on the right-hand side above, we obtain after some straightforward algebraic simplification:

$$
\begin{aligned}
& \hat{g}(\mathbf{x})-g(\mathbf{x}) \\
& =\Gamma\left(\mathbf{x}, \overline{\mathbf{X}}_{t} ; p_{t}, q_{t}\right) D^{-1}\left(p_{t}, q_{t}, t\right) \sum_{\mathbf{X}_{i} \in t} \Gamma^{T}\left(\mathbf{X}_{i}, \overline{\mathbf{X}}_{t} ; p_{t}, q_{t}\right)\left\{g\left(\mathbf{X}_{i}\right)\right. \\
& \quad-g(\mathbf{x})\}+\Gamma\left(\mathbf{x}, \overline{\mathbf{X}}_{t} ; p_{t}, q_{t}\right) D^{-1}\left(p_{t}, q_{t}, t\right) \sum_{\mathbf{X}_{i} \in t} \Gamma^{T} \\
& \quad \times\left(\mathbf{X}_{i}, \overline{\mathbf{X}}_{t} ; p_{t}, q_{t}\right) \epsilon_{i} .
\end{aligned}
$$


This is a consequence of $D^{-1}\left(p_{t}, q_{t}, t\right) \sum_{\mathbf{X}_{i t}} \Gamma^{T}\left(\mathbf{X}_{i}, \overline{\mathbf{X}}_{t}\right.$; $\left.p_{t}, q_{t}\right)$ being an $s(\mathbf{U})$-dimensional vector with a one as the first coordinate and the other coordinates all equal to zero.

Now, following the ideas in the proof of Theorem 1 in Chaudhuri et al. (1994), the first term on the right-hand side in the expression for $\hat{g}(\mathbf{x})-g(\mathbf{x})$ can be viewed as a bias term while the second term can be thought of as a variance term that occurs in the decomposition of the error in a nonparametric regression estimate. Since the function $g(\mathbf{x})$ is a uniformly continuous function in $C$, it follows immediately that the bias term tends to zero in probability uniformly in $\mathbf{x}$ as the sample size grows to infinity. Furthermore, since the partition sets are hyper-rectangles and the moment generating function of $Y-g(\mathbf{X})$ is bounded, the arguments in the proof of Chaudhuri et al. (1994, Theorem 1) imply that the variance term tends to zero in probability uniformly in $\mathbf{x}$.

\section{Conclusions}

We set out seeking an algorithm that can automatically generate interpretable and visualizable models with good prediction accuracy. We gave as motivation the difficulty of interpreting the coefficients in a multiple linear model. Our solution embraces rather than discards the linear model, but we limit it to at most two regressors and apply it to partitions of the data. As the Boston and baseball examples demonstrate, this approach can yield models that fit the data at least as well as those built by human experts. Our models do not require special training or equipment for visualization; all that is needed are tree diagrams, graphs, and contour maps. Our trees are also substantially more compact than piecewise constant trees. And they can be interpreted without worrying about selection bias.

In terms of prediction accuracy, our piecewise tworegressor model Gf 2 yields on average about $80 \%$ of the prediction MSE of least squares linear regression. Although Gf 2 does not have the lowest average, its prediction MSE does not differ significantly from the lowest at the 0.05 simultaneous level of significance. We note that the lowest average value over the 27 algorithms is $60 \%$. If the datasets used in our study are representative of all real datasets, this result suggests that it is hard to beat the prediction MSE of least squares linear regression by a very large amount in real applications. Given this, it is quite reasonable to demand more from all algorithms, including interpretability and visualizability of their models.

\section{Acknowledgements}

The authors are grateful to two referees for their comments. Kim's research was partially supported by grant R01-2005-000-11057-0 from the Basic Research Program of the Korea Science and Engineering Foundation. Loh's research was partially supported by the National Science Foundation under grant DMS-0402470 and by the US Army Research Laboratory and the US Army Research Office under grant W911NF-05-1-0047. Shih's research was partially supported by a grant from the National Science Council of Taiwan.

\section{References}

Aaberge, R., Colombino, U. and Strom, S. (1999) Labor supply in Italy: an empirical analysis of joint household decisions, with taxes and quantity constraints. Journal of Applied Econometrics, 14, 403-422.

Afifi, A. and Azen, S. (1979) Statistical Analysis: a Computer Oriented Approach, 2nd edn., Academic Press, New York, NY.

Belsley, D.A., Kuh, E. and Welsch, R.E. (1980) Regression Diagnostics: Identifying Influential Data and Sources of Collinearity, Wiley, New York, NY.

Berndt, E.R. (1991) The Practice of Econometrics, Addison-Wesley, New York, NY.

Blake, C. and Merz, C. (1998) UCI repository of machine learning databases. http://www.ics.uci.edu/mlearn/MLRepository.html.

Bollino, C.A., Perali, F. and Rossi, N. (2000) Linear household technologies. Journal of Applied Econometrics, 15, 253-274.

Box, G.E.P. (1979) Robustness in the strategy of scientific model building, in Robustness in Statistics, Launer, R.L. and Wilkinson, G.N. (eds.), Academic Press, New York, NY. pp. 201-236.

Breiman, L. and Friedman, J. (1988) Estimating optimal transformations for multiple regression and correlation. Journal of the American Statistical Association, 83, 580-597.

Breiman, L., Friedman, J. H., Olshen, R. A. and Stone, C. J. (1984) Classification and Regression Trees, Wadsworth, Belmont, MA.

Bryant, P.G. and Smith, M.A. (1996) Practical Data Analysis: Case Studies in Business Statistics, Volume 3, Irwin/McGraw Hill, Boston, MA.

Chattopadhyay, S. (2003) Divergence in alternative Hicksian welfare measures: the case of revealed preference for public amenities. Journal of Applied Econometrics, 17, 641-666.

Chaudhuri, P., Huang, M.-C., Loh, W.-Y. and Yao, R. (1994) Piecewisepolynomial regression trees. Statistica Sinica, 4, 143-167.

Chu, S. (2001) Pricing the C's of diamond stones. Available at http://www.amstat.org/publications/jse.

Cochran, J.J. (2000) Career records for all modern position players eligible for the major league baseball hall of fame. Available at http://www.amstat.org/publications/jse.

Cochran, J. J. (2002) Data management, exploratory data analysis, and regression analysis with 1969-2000 major league baseball attendance. Available at http://www.amstat.org/publications/jse.

Cook, D. (1998) Regression Graphics: Ideas for Studying Regression Through Graphics, Wiley, New York.

Cook, D. and Weisberg, S. (1994) An Introduction to Regression Graphics, Wiley, New York, NY.

Deb, P. and Trivedi, P.K. (1997) Demand for medical care by the elderly: a finite mixture approach. Journal of Applied Econometrics, 12, 313336.

Delgado, M.A. and Mora, J. (1998) Testing non-nested semiparametric models: an application to Engel curves specification. Journal of Applied Econometrics, 13, 145-162.

Denman, N. and Gregory, D. (1998) Analysis of sugar cane yields in the Mulgrave area, for the 1997 sugar cane season. Technical report, MS305 Data Analysis Project, Department of Mathematics, University of Queensland, Queensland, Australia.

Fernandez, C., Ley, E. and Steel, M.F.J. (2002) Bayesian modelling of catch in a north-west Atlantic fishery. Applied Statistics, 51, 257 280 . 
Friedman, J. (1991) Multivariate adaptive regression splines (with discussion). Annals of Statistics, 19, 1-141.

Friedman, J. (2001) Greedy function approximation: a gradient boosting machine. Annals of Statistics, 29, 1189-1232.

Friedman, J.H. and Stuetzle, W. (1981) Projection pursuit regression. Journal of the American Statistical Association, 76, 817-823.

Gilley, O.W. and Pace, R.K. (1996) On the Harrison and Rubinfeld data. Journal of Environmental Economics and Management, 31, 403-405.

Hair, J.F., Anderson, R.E., Tatham, R.L. and Black, W.C. (1998) Multivariate Data Analysis, Prentice Hall, Upper Saddle River, NJ.

Hallin, M. and Ingenbleek, J.-F. (1983) The Swedish automobile portfolio in 1977: a statistical study. Scandinavian Actuarial Journal, 83, 49-64.

Harrell, Jr., F. E. (2001) Regression Modeling Strategies: With Applications to Linear Models, Logistic Regression, and Survival Analysis, Springer-Verlag, New York, NY.

Harrison, D. and Rubinfeld, D.L. (1978) Hedonic prices and the demand for clean air. Journal of Environmental Economics and Management, $\mathbf{5}, 81-102$.

Hastie, T. and Tibshirani, R. (1990) Generalized Additive Models, CRC Press, Boca Raton, FL.

Hoaglin, D.C. and Velleman, P.F. (1995) A critical look at some analyses of major league baseball salaries. American Statistician, 49, 277-285.

Horrace, W.C. and Schmidt, P. (2000) Multiple comparisons with the best, with economic applications. Journal of Applied Econometrics, $15,1-26$.

Huber, P. J. (1981) Robust Statistics, Wiley, New York, NY.

Kenkel, D.S. and Terza, J.V. (2001) The effect of physician advice on alcohol consumption: Count regression with an endogenous treatment effect. Journal of Applied Econometrics, 16, 165-184.

Kooperberg, C., Bose, S. and Stone, C. (1997) Polychotomous regression. Journal of the American Statistical Association, 92, 117-127.

Laroque, G. and Salanie, B. (2002) Labor market institutions and employment in France. Journal of Applied Econometrics, 17, 25-28.

Liu, Z. and Stengos, T. (1999) Non-linearities in cross country growth regressions: a semiparametric approach. Journal of Applied Econometrics, 14, 527-538.

Loh, W.-Y. (2002) Regression trees with unbiased variable selection and interaction detection. Statistica Sinica, 12, 361-386.

Lutkepohl, H., Terasvirta, T. and Wolters, J. (1999) Investigating stability and linearity of a German M1 money demand function. Journal of Applied Econometrics, 14, 511-525.

Martins, M.F.O. (2001) Parametric and semiparametric estimation of sample selection models: an empirical application to the female labour force in Portugal. Journal of Applied Econometrics, 16, 23-40.

Miller, A. (2002) Subset Selection in Regression, 2nd edn., Chapman \& Hall, Boca Raton, FL.

Neter, J., Kutner, M.H., Nachtsheim, C.J. and Wasserman, W. (1996) Applied Linear Statistical Models, 4th edn., Irwin, Boston, MA.

Olson, C.A. (1998) A comparison of parametric and semiparametric estimates of the effect of spousal health insurance coverage on weekly hours worked by wives. Journal of Applied Econometrics, 13, 543565.

Onoyama, K., Ohsumi, N., Mitsumochi, N. and Kishihara, T. (1998) Data analysis of deer-train collisions in eastern Hokkaido, Japan, in Data Science, Classification, and Related Methods, Hayashi, C.,
Ohsumi, N., Yajima, K., Tanaka, Y., Bock, H.-H. and Baba Y. (eds.), Springer-Verlag, Tokyo, Japan, pp. 746-751.

Pace, R.K. and Barry, R. (1997) Sparse spatial autoregressions. Statistics and Probability Letters, 33, 291-297.

Penrose, K., Nelson, A. and Fisher, A. (1985) Generalized body composition prediction equation for men using simple measurement techniques. Medicine and Science in Sports and Exercise, 17, 189.

Quinlan, J.R. (1992) Learning with continuous classes, in Proceedings of the 5th Australian Joint Conference on Artificial Intelligence, World Scientific, Singapore, pp. 343-348.

Rawlings, J.O. (1988) Applied Regression Analysis: A Research Tool, Wadsworth \& Brooks/Cole.

Schafgans, M.M. (1998) Ethnic wage differences in Malaysia: parametric and semiparametric estimation of the Chinese-Malay wage gap. Journal of Applied Econometrics, 13, 481-504.

Simonoff, J. (1996) Smoothing Methods in Statistics, Springer-Verlag, New York, NY.

Steinberg, D. and Colla, P. (1995) CART: Tree-Structured NonParametric Data Analysis, Salford Systems, San Diego, CA.

Torgo, L. (1999) Inductive learning of tree-based regression models, $\mathrm{PhD}$ thesis, Department of Computer Science, Faculty of Sciences, University of Porto, Porto, Portugal.

Venables, W.N. and Ripley, B.D. (1999) Modern Applied Statistics with S-Plus, Springer-Verlag, New York, NY.

Weiss, S. and Indurkhya, N. (1995) Rule-based machine learning methods for functional prediction. Journal of Artificial Intelligence Research, 3, 383-403.

Witten, I. and Frank, E. (2000) Data Mining: Practical Machine Learning Tools and Techniques with JAVA Implementations, Morgan Kaufmann, San Francisco, CA.

\section{Biographies}

Hyunjoong Kim is an Assistant Professor in the Department of Applied Statistics, Yonsei University, Korea. He has a $\mathrm{PhD}$ in Statistics from the University of Wisconsin, Madison. His research interests lie in data mining, tree-based statistical modeling, and statistical computing.

Wei-Yin Loh is a Professor in the Department of Statistics, University of Wisconsin, Madison. He has a PhD in Statistics from the University of California, Berkeley. His research interests lie in statistical theory and methodology, including data mining and machine learning techniques.

Yu-Shan Shih is a Professor in the Department of Mathematics, National Chung Cheng University, Taiwan, ROC. He has a $\mathrm{PhD}$ in Statistics from the University of Wisconsin, Madison. His research interests lie in multivariate methods, computational statistics and tree-structured methods.

Probal Chaudhuri is a Professor in the Theoretical Statistics and Mathematics Unit, Indian Statistical Institute, Calcutta, India. He has a PhD degree in Statistics from the University of California, Berkeley. His research interests include nonparametric statistics, robustness and applications of statistics in engineering and biological sciences. He is a Fellow of the Indian Academy of Sciences. 
Copyright of IIE Transactions is the property of Taylor \& Francis Ltd and its content may not be copied or emailed to multiple sites or posted to a listserv without the copyright holder's express written permission. However, users may print, download, or email articles for individual use. 